\title{
Superconducting correlations induced by charge ordering in cuprate superconductors and Fermi arc formation
}

\author{
E.V.L. de Mello ${ }^{1, \text {, }}$ and J.E. Sonier ${ }^{2,3}$ \\ ${ }^{1}$ Instituto de Física, Universidade Federal Fluminense, 24210-346 Niterói, RJ, Brazil \\ ${ }^{2}$ Department of Physics, Simon Fraser University, Burnaby, British Columbia V5A 1S6, Canada. \\ ${ }^{3}$ Canadian Institute for Advanced Research, Toronto, Ontario M5G 1Z8, Canada.
}

\begin{abstract}
We have developed a generalized electronic phase separation model of high-temperature cuprate superconductors that links the two distinct energy scales of the superconducting (SC) and pseudogap (PG) phases via a charge-density-wave (CDW) state. We show that simulated electronic-density modulations resembling the charge order $(\mathrm{CO})$ modulations detected in cuprates intertwine the SC and charge orders by localizing charge and providing the energy scale for a spatially periodic SC attractive potential. Bulk superconductivity is achieved with the inclusion of Josephson coupling between nanoscale domains of intertwined fluctuating CDW and SC orders, and local SC phase fluctuations give rise to the Fermi arcs along the nodal directions of the SC gap. We demonstrate the validity of the model by reproducing the hole-doping dependence of the $\mathrm{PG}$ onset temperature $T^{*}$, and the $\mathrm{SC}$ transition temperature $T_{c}$ of $\mathrm{YBa}_{2} \mathrm{Cu}_{3} \mathrm{O}_{\mathrm{y}}$ and $\mathrm{Bi}_{2-y} \mathrm{~Pb}_{\mathrm{y}} \mathrm{Sr}_{2-\mathrm{z}} \mathrm{La}_{\mathrm{z}} \mathrm{CuO}_{6+\delta}$. The results show that the periodicity of the CDW order is controlled by the PG energy scale, and the hole-doping dependence of the SC energy gap is controlled by the charge ordering free energy.
\end{abstract}

\section{INTRODUCTION}

Experiments using different methods have established the occurrence of short-range, incommensurate static CDW correlations in a variety of high-temperature SC cuprates $-1-17$. With the exception of La-based cuprates in which CDW order is accompanied by spin order, the charge order (CO) observed in different cuprate families appears to be similar. In zero magnetic field, the CDW order is essentially two-dimensional. The wave vector of the CDW order is parallel to the $\mathrm{Cu}$ $\mathrm{O}$ bond directions along the $\mathrm{a}$ and $\mathrm{b}$ axes, and decreases in magnitude with increased charge doping. While much of the experimental data cannot distinguish between checkerboard (bidirectional) or alternating stripe (unidirectional) $\mathrm{CO}$, recent resonant $\mathrm{X}$-ray scattering (RXS) experiments on underdoped $\mathrm{YBa}_{2} \mathrm{Cu}_{3} \mathrm{O}_{7}(\mathrm{Y} 123)^{\underline{7}}$ and an analysis of scanning tunneling microscopy (STM) data for $\mathrm{Bi}_{2} \mathrm{Sr}_{2} \mathrm{CaCu}_{2} \mathrm{O}_{8+\delta^{18}}$ indicate that the inter-unit-cell character is one of segregated or overlapping unidirectional charge-ordered stripes. Furthermore, it has been found that the CDW order possesses a $d$ wave intra-unit-cell symmetry with the modulated charge primarily on the $\mathrm{O}-2 p$ orbitals linking the $\mathrm{Cu}$ atoms $-\underline{-10}$. Since the SC order parameter also has $d$-wave symmetry, this local charge or bond order symmetry supports theoretical proposals that suggest the charge and SC order parameters are intimately intertwined. Some attribute the $d$-wave CO symmetry to quasiparticle scattering by antiferromagnetic (AF) fluctuations near a metallic quantum critical point, which also gives rise to the d-wave superconductivity $\underline{\underline{19-21}}$. Alternatively, it has been proposed that CDW order in cuprates is a consequence of a pair-density wave (PDW) phase, in which the SC order parameter is periodically modulated in space due to the Cooper pairs having finite momentum²-25.

The aim of our work is to establish a quantitative link between the inter-unit-cell dependence of the $\mathrm{CO}$ resolved by RXS and imaged in real space by STM, and the energy gaps of the PG and SC phases. Our model is based on an intrinsic propensity for mesoscale electronic phase separation be- low an onset temperature $T_{\mathrm{PS}}$ that follows the hole-doping dependence of the PG temperature $T^{*}$. This picture is similar to that previously advocated by Fradkin and Kivelson ${ }^{26}$. We presume the onset of fluctuating CDW order domains at $T^{*}$, where STM measurements on $\mathrm{Bi}_{2} \mathrm{Sr}_{2} \mathrm{CaCu}_{2} \mathrm{O}_{8+\delta}$ have detected the emergence of charge stripes that extend into the overdoped regime ${ }^{27}$. The short-range static $\mathrm{CO}$ that has been observed by X-rays at a lower temperature $T_{\mathrm{CO}} \leq T^{*}$ is assumed to be confined to local regions where fluctuating CDW order has become pinned by disorder. Contrary to this assumption, we note that in $\mathrm{HgBa}_{2} \mathrm{CuO}_{4+\delta}(\mathrm{Hg} 1201) \mathrm{CDW}$ order observed by X-ray scattering vanishes already well below optimal doping 28 . This seems to be due to the presence of pairs of interstitial oxygens within the same unit cell specific to $\mathrm{Hg} 1201$. Although not captured by our model, it is also important to recognize that the pseudogap region marks the onset of an intra-unit-cell magnetic order ${ }^{29.30}$, a true phase transition that modifies ultrasonic waves ${ }^{31}$, an increase in antiferromagnetic correlations ${ }^{32}$ and global inversion-symmetry breaking 33 .

Another important ingredient of our model is the experimental observation that the CDW periodicity is independent of temperature, leading us to surmise that the CDW periodicity is set by the onset of the PG at $T^{*}$. This infers that the CDW order is a consequence of the PG formation. At low doping $(p \leq 0.12)$ where $T_{\mathrm{CO}}$ decreases with decreasing doping, CDW order is potentially suppressed by a slowing down of spin fluctuations and a tendency toward static SDW order. Compatible with experimental signatures of pairing or SC correlations persisting above $T_{C} \stackrel{34}{=}$, our model shows that CDW order in the PG regime may induce SC domains that grow and connect to establish bulk superconductivity at $T_{c}$.

\section{SIMULATION OF THE CHARGE-ORDERED STATE}

Our approach is to first simulate spatial modulations of the electronic structure resembling experimentally resolved inter- 

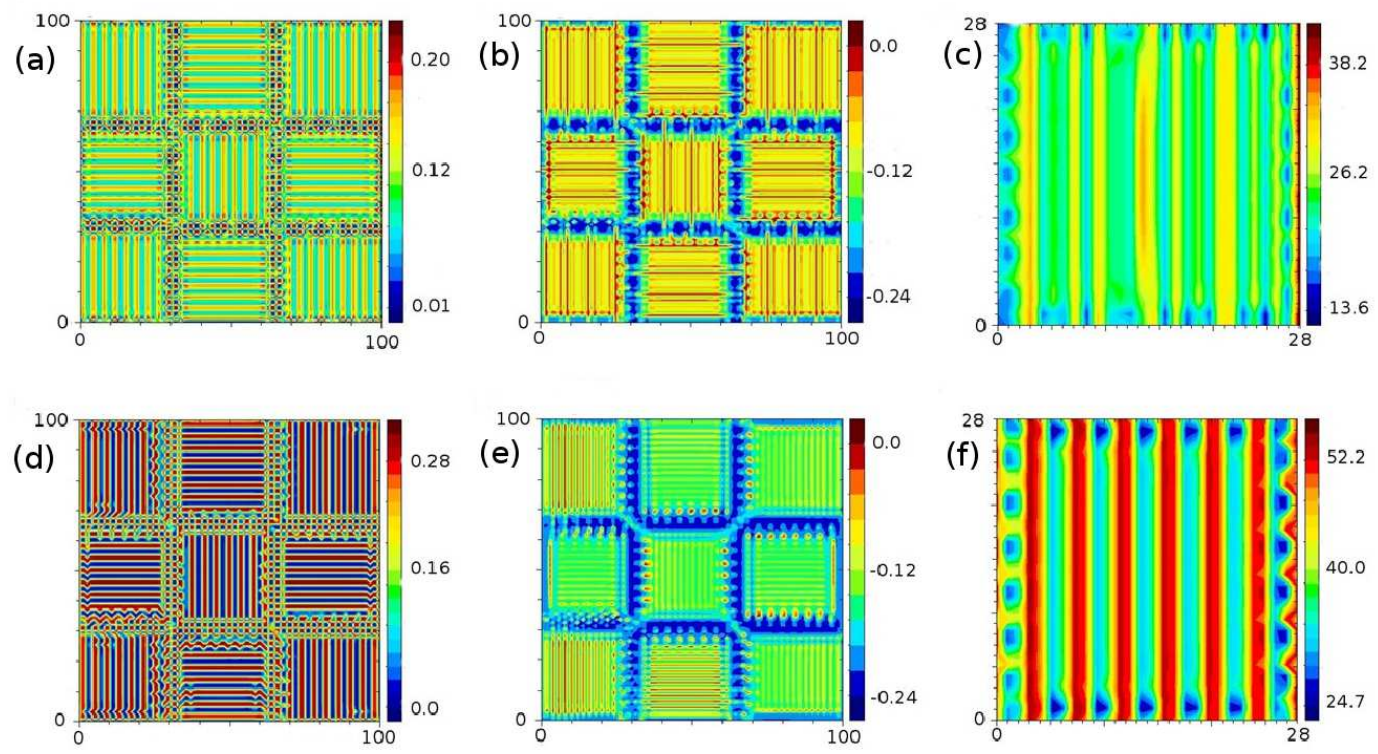

FIG. 1. Contour plots of the electronic density $p(\mathbf{r})$ calculated on a square lattice of $100 \times 100$ unit cells, with average charge densities of $p=0.12$ in (a) and $p=0.16$ in (d). The charge order wavelengths are $\lambda_{\mathrm{CO}}=3.15 a_{0}$ (a) and $\lambda_{\mathrm{CO}}=3.49 a_{0}$ in (d), corresponding to the charge order wave vectors determined by momentum-resolved X-ray probes ${ }^{4.5 .7}$. (b), (e) Corresponding spatial dependence of the free-energy potential $V_{\mathrm{GL}}(\mathbf{r})$. The periodicity of the potential manifests in the periodic modulations of the charge density. (c), (f) Results of calculations of the d-wave pairing potential $\Delta_{d}(\mathbf{r})$ displayed for a single domain over a $28 \times 28$ unit cell area (in meV unit). The spatial average value of the pair potential $\left\langle\Delta_{d}(\mathbf{r})\right\rangle$ is $25.5 \mathrm{meV}$ at $p=0.12$ in (c), and $43.8 \mathrm{meV}$ at $p=0.16$ in (f).

unit-cell CO modulations, using the time-dependent CahnHilliard $(\mathrm{CH})$ differential equation $\stackrel{41}{*}$. Besides generating the desired $\mathrm{CDW}$ order, the $\mathrm{CH}$ approach yields the associated free-energy modulations, which we assume scales with a periodic attractive potential in the subsequent SC calculations. The starting point is the introduction of a time-dependent conserved order parameter associated with the local electronic density, $u(\mathbf{r}, t)=[p(\mathbf{r}, t)-p] / p$, where $p$ is the average charge density and $p(\mathbf{r}, t)$ is the charge density at a position $\mathbf{r}$ in the plane. The Ginzburg-Landau (GL) free energy density of the system is of the form

$$
f(u)=\frac{1}{2} \varepsilon|\nabla u|^{2}+V_{G L}(u, T),
$$

where $V_{\mathrm{GL}}(u, T)=-\alpha\left[T_{\mathrm{PS}}-T\right] u^{2} / 2+B^{2} u^{4} / 4+\ldots$ is a double-well potential that characterizes the electronic phase separation below $T_{\mathrm{PS}}$. The parameters $\alpha$ and $B$ are constants, and $\varepsilon$ controls the spatial separation of the charge-segregated patches. The $\mathrm{CH}$ equation obtained from the continuity equation for the local free energy current density $\mathrm{J}=-\mathrm{M} \nabla \mu$ (where $\mathbf{M}$ is the charge mobility and $\mu=\partial f / \partial \mu$ is the chemical potential). Its solution is described in the Apendix I on charge ordering simulations. For each time step the $\mathrm{CH}$ equation is solved for $u(\mathbf{r}, t)$, and $p(\mathbf{r}, t)$ is obtained. We adjust the parameters of the free energy such that when the periodicity of $p(\mathbf{r}, t)$ matches that of the experimentally observed CDW order, the calculation is stopped and the solution is taken to be the spatially-dependent static electronic density $p(\mathbf{r})$. Since the method described here does not generate an intra-unit-cell
CO symmetry, it is applicable to systems that have SC pairing and $\mathrm{CO}$ symmetries other than d-wave.

Figure 1(a) shows a simulation of alternating planar domains of $90^{\circ}$-rotated charge stripes with an intra-domain periodicity compatible with RXS data for detwinned Y123 at $p=0.12[8]$. Within each domain the $\mathrm{CO}$ wavelength is $\lambda_{\mathrm{CO}}=3.15 a_{0}$, where the in-plane lattice constant is $a_{0}=$ $3.85 \AA$. The $\mathrm{CO}$ wavelength in Y123 measured by various $\mathrm{X}$ ray methods increases with increased hole doping $4.5,42$. We have used such data to generate similar $\mathrm{CO}$ striped patterns for Y123 at $p=0.16$ (Fig. 11d)) and $p=0.09$.

STM differential conductance maps for optimally-doped $\left(T_{c}=35 \mathrm{~K}\right)$ and underdoped $\left(T_{c}=32 \mathrm{~K}\right.$ and $\left.T_{c}=25 \mathrm{~K}\right)$ $\mathrm{Bi}_{2-y} \mathrm{~Pb}_{\mathrm{y}} \mathrm{Sr}_{2-\mathrm{z}} \mathrm{La}_{\mathrm{z}} \mathrm{CuO}_{6+\delta}[(\mathrm{Pb}, \mathrm{La})-\mathrm{Bi} 2201]$ samples 11,12 exhibit checkerboard patterns (indicative of the simultaneous presence of both CDW domains) with $6.20 .2 a_{0}, 5.10 .2 a_{0}$, and 4.50.2 $a_{0}$ unit cell $\left(a_{0}=3.83 \AA\right)$ periodicity, respectively. The increase in the $\mathrm{CO}$ wavelength with increased hole doping agrees well with X-ray scattering and STM measurements on Bi2201 without cation substitutions ${ }^{4}$. We have used the following formula from Ref. 43 to calculate the average number of holes per $\mathrm{Cu}$ for the (Pb, La)-Bi2201 samples in Ref. 11: $T_{c} / T_{c}^{\max }=1-250(p-0.16)^{2}$, where $T_{c}^{\max }=35 \mathrm{~K}$. This calculation yields $p=0.126$ and $p=0.141$ for the two underdoped samples. Figs. 2 (a) and 2 d) display $\mathrm{CO}$ checkerboard patterns simulated by the $\mathrm{CH}$ equation that resemble the STM differential conductance maps for $(\mathrm{Pb}, \mathrm{La})-\mathrm{Bi} 2201$ at $p=0.126$ and $p=0.16$.

The CO periodicity is manifest in the spatial dependence of the free-energy potential $V_{\mathrm{GL}}(\mathbf{r})$, shown in Figs. 1 1 b) and 

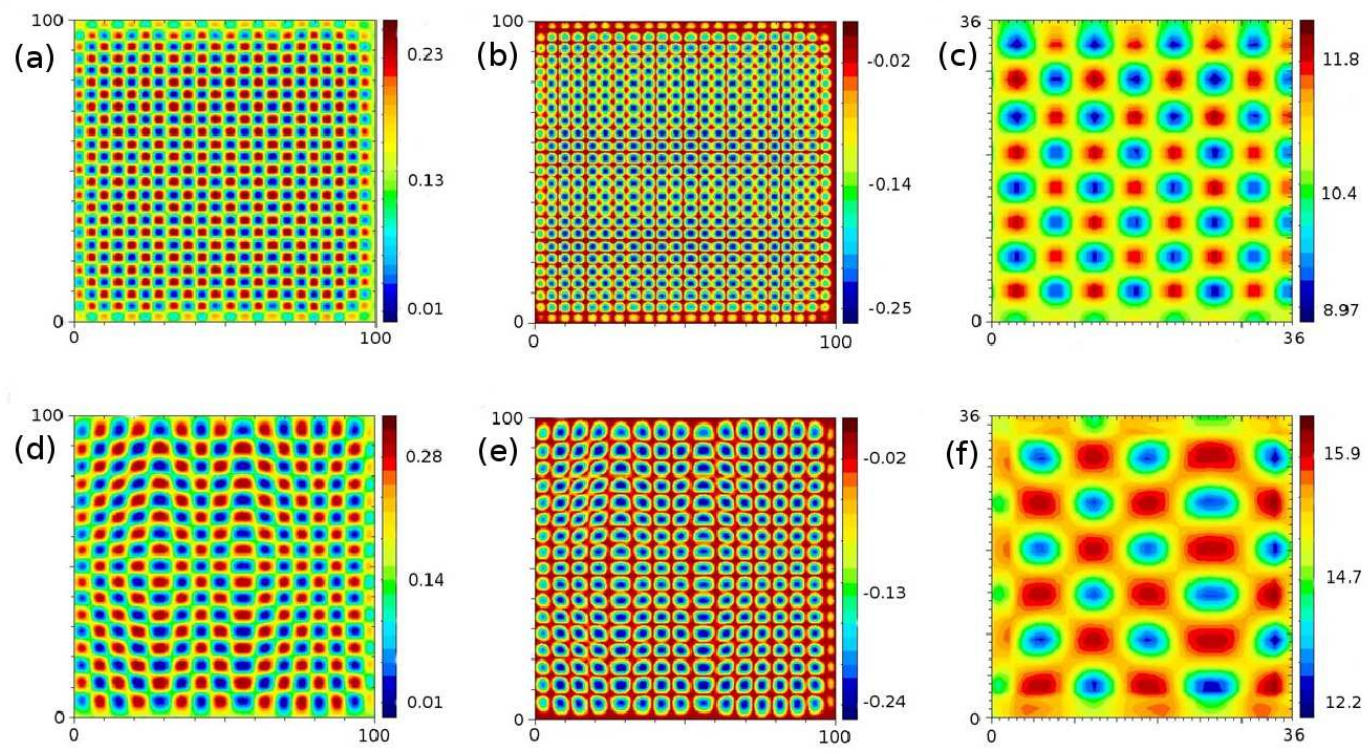

FIG. 2. Contour plots of the electronic density $p(\mathbf{r})$ calculated on a square lattice of $100 \times 100$ unit cells, assuming average charge densities of $p=0.126$ in (a) and $p=0.16$ in (d). The charge order wavelengths are $\lambda_{\mathrm{CO}}=4.5 a_{0}$ in a and $\lambda_{\mathrm{CO}}=6.2 a_{0}$ in (d), matching the checkerboard wavelength of the STM conductance maps of underdoped $\left(T_{c}=25 \mathrm{~K} ; p=0.126\right)$ and optimally-doped (Pb, La)-Bi2201 in Ref. 11. (b), (e), Corresponding spatial dependence of the free-energy potential $V_{\mathrm{GL}}(\mathbf{r})$. (c), (f), Results of calculations of the $d$-wave pairing potential $\Delta_{d}(\mathbf{r})$ displayed over a $36 \times 36$ unit cell area (in meV unit). The spatial average value of the pair potential $\left\langle\Delta_{d}(\mathbf{r})\right\rangle$ is $9.2 \mathrm{meV}$ at $p=0.126$ in (c), and $15.8 \mathrm{meV}$ at $p=0.16$ in (f).

1(e), and Figs. 2) b) and2 (e). The central assumption in our model is that by confining charge, a fluctuating CDW periodic potential that scales with $V_{\mathrm{GL}}(\mathbf{r})$ mediates the attractive two-body SC interaction. In particular, we assume the fluctuating periodic potential has the same periodicity as the static CO detected experimentally and has a time-averaged potential well depth that is proportional to the depth of the static periodic potential. In what follows, we make the approximation $\left\langle V_{\mathrm{GL}}(\mathbf{r})\right\rangle$, where $\left\langle V_{\mathrm{GL}}(\mathbf{r})\right\rangle$ is the spatial average of $V_{\mathrm{GL}}(\mathbf{r})$ over a $100 \times 100$ unit cell area.

\section{SUPERCONDUCTING CALCULATIONS IN THE CHARGE-ORDERED STATE}

Next we use the free-energy simulations and experimentally determined input parameters for the optimally-doped compounds to deduce the SC energy gap $\Delta_{\mathrm{SC}}$, the pseudogap $\Delta_{\mathrm{PG}}, T_{c}$ and $T^{*}$ for the underdoped samples. To derive the local SC gap we solved the Bogoliubov-deGennes (BdG) equations via self-consistent calculations based on a Hubbard Hamiltonian [Eq. B1]. The calculations were performed for a sub-lattice about the center of the simulated charge density maps, using periodic boundary conditions and governed by self-consistent conditions for a spatially-varying d-wave pairing potential $\Delta_{d}(\mathbf{r})$ and hole density $p(\mathbf{r})$ [Eq. B4) and B5]. We find that the spatial-average $\left\langle\Delta_{d}(\mathbf{r})\right\rangle$ decreases with a reduction of $p$ (below $p=0.16$ ), but increases with decreasing $\lambda_{\mathrm{CO}}$. The latter behavior is because as the two holes are forced closer together by the narrower confining potential the binding energy of the two-body interaction increases. The results on the $\mathrm{CuO}_{2}$ plane shown in Figs. 1(c), 1(f), 2(c) and 2(f) indicate that in our approach the PDW is a consequence of the CDW.

The values of $\left\langle V_{\mathrm{GL}}(\mathbf{r})\right\rangle$ at optimal doping were multiplied by a scaling factor, such that the calculations (Figs. 1ff) and 2 ff) generate an average value of the pairing potential $\left\langle\Delta_{d}(\mathbf{r})\right\rangle$ for $p=0.16$ that is close to the experimentally estimated value of the low-temperature SC gap $\Delta_{\mathrm{SC}}$. To calculate $\left\langle\Delta_{d}(\mathbf{r})\right\rangle$ for the underdoped samples (Tables $\square$ and $\Pi$, this same scaling factor was subsequently applied to the respective values of $\left\langle V_{\mathrm{GL}}(\mathbf{r})\right\rangle$. For $(\mathrm{Pb}, \mathrm{La})-\mathrm{Bi} 2201$, the value of $\left\langle V_{\mathrm{GL}}(\mathbf{r})\right\rangle$ varies little with doping, and hence $p$ and $\lambda_{\mathrm{CO}}$ are responsible for the hole-doping dependence of $\left\langle\Delta_{d}(\mathbf{r})\right\rangle$. Experimental estimates of the SC gap for the $p=0.126$ and 0.141 samples are not reported, but the calculated values of $\left\langle\Delta_{d}(\mathbf{r})\right\rangle$ for the underdoped samples (Table II) roughly follow the trend expected if the ratio $\Delta_{\mathrm{SC}} / k_{\mathrm{B}} T_{c}$ is independent of $p$. In contrast to $(\mathrm{Pb}, \mathrm{La})-\mathrm{Bi} 2201$, the doping dependence of $\lambda_{\mathrm{CO}}$ in Y123 is weaker, and the $\mathrm{CH}$ simulations of charge stripes are characterized by a significant change in $\left\langle V_{\mathrm{GL}}(\mathbf{r})\right\rangle$ with doping (Fig 4). Consequently, the depth of the periodic potential plays an important role in the calculation of the doping dependence of $\left\langle\Delta_{d}(\mathbf{r})\right\rangle$ for Y123. The calculated values of $\left\langle\Delta_{d}(\mathbf{r})\right\rangle$ at $\mathrm{p}=0.09$ and 0.16 agree well with an empirical relation for $\Delta_{\mathrm{SC}}(p)$ that describes a number of high- $T_{c}$ cuprate superconductors 44 . The calculated result at $p=0.12$ falls below this universal curve, which is consistent with the well-known plateau of $T_{c}(p)$ for Y123 near 1/8 hole doping.

An estimate of $T_{c}$ is obtained by self-consistently solving 

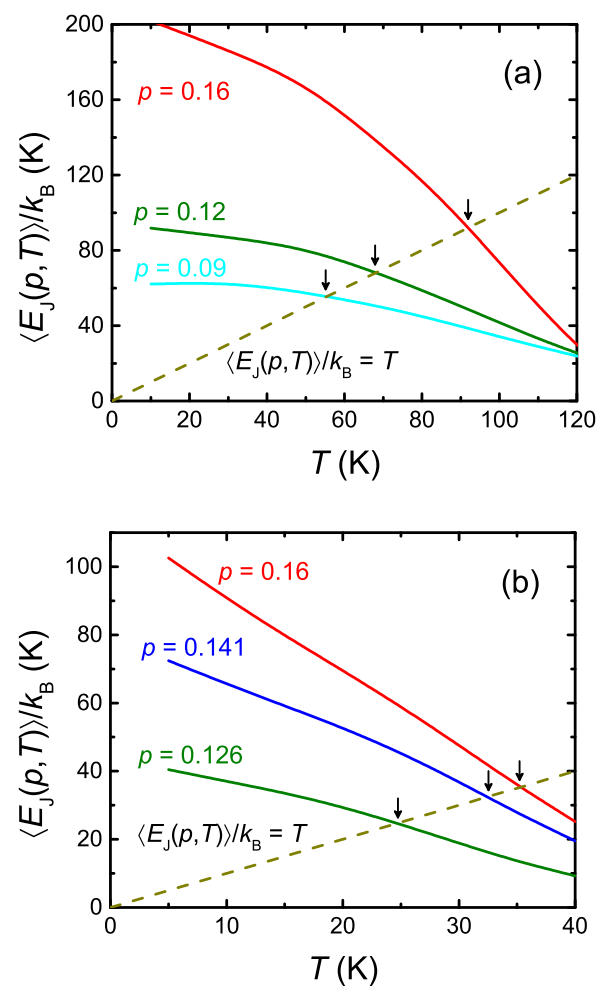

FIG. 3. (Color online) Calculated values of the superconducting transition temperature. $(\mathrm{a}, \mathrm{b})$ The temperature dependence of the average Josephson coupling energy $\left\langle E_{\mathrm{J}}(p, T)\right\rangle$ (divided by Boltzmann constant $k_{\mathrm{B}}$ ) for $\mathrm{Y} 123$ at $p=0.09,0.12$, and 0.16 in (a), and (Pb, La)-Bi2201 at $p=0.126,0.141$, and 0.16 in (b). The values of $T_{c}$ correspond to the intersections of the dashed straight line $\left\langle E_{\mathrm{J}}(p, T)\right\rangle / k_{\mathrm{B}}=T$ with the $\left\langle E_{\mathrm{J}}(p, T)\right\rangle / k_{\mathrm{B}}$ curves and are marked by the arrows.

the BdG equations with a temperature-dependent GL potential

$$
V(T, p)=V(0, p)\left[1-T / T_{\mathrm{PS}}(p)\right]^{2},
$$

where we take $V(0, p) \approx\left\langle V_{\mathrm{GL}}(\mathbf{r}, p)\right\rangle$ and $T_{\mathrm{PS}}(p)$, the onset of phase separation transition, is taken to be equan to $T^{*}(p)$ in the calculations. Because of the $\mathrm{BdG}$ approach and the above equation, the value of $\left\langle\Delta_{d}(\mathbf{r}, T)\right\rangle$ decreases with increasing temperature, but the it remains finite in many regions of the system for a significant range of temperature above Tc. This is consistent with the body of experimental results on cuprates mentioned earlier that are suggestive of persisting SC correlations above $T_{c}{ }^{34-40}$. Typical $\left\langle\Delta_{d}(\mathbf{r}, T)\right\rangle$ plots of three Y123 compounds are shown in Fig. A2. Next we assume that bulk superconductivity is achieved via Josephson coupling between different closely spaced patches of intertwined $\mathrm{CO}$ and $\mathrm{SC}$ pairing. We assume there is SC phase coherence within the patches, and that there are many such closely spaced SC domains slightly above $T_{c}(p)$ forming junctions with an average tunnel y proportional to the normal-state resistance immediately above $T_{c}(p)$. As explained previously ${ }^{45}$, for a $d$-wave superconductor in single-crystal form it is sufficient to use the

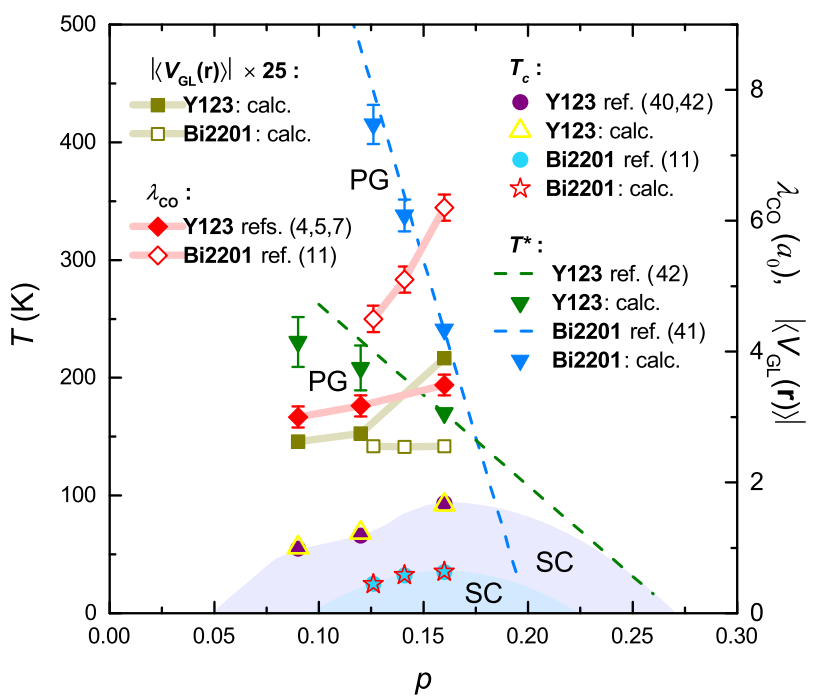

FIG. 4. Comparison of experimental and calculated values of $T_{c}$ and $T^{*}$ versus hole doping. Also shown is the experimentally determined doping dependence of the charge order wavelength $\lambda_{\mathrm{CO}}$ for both compounds, as well as the doping dependence of the calculated absolute value of the spatial average of the free-energy potential $\left|\left\langle V_{\mathrm{GL}}(\mathbf{r})\right\rangle\right|$. For display purposes $\left|\left\langle V_{\mathrm{GL}}(\mathbf{r})\right\rangle\right|$ is shown multiplied by a factor of 25 .

following relation for the average Josephson coupling energy

$$
\left\langle E_{\mathrm{J}}(p, T)\right\rangle=\frac{\pi \hbar\left\langle\Delta_{d}(\mathbf{r}, T)\right\rangle}{2 e^{2} R_{\mathrm{n}}(p)} \tanh \left[\frac{\left\langle\Delta_{d}(\mathbf{r}, T)\right\rangle}{2 k_{\mathrm{B}} T}\right]
$$

where $\left\langle\Delta_{d}(\mathbf{r}, T)\right\rangle=\sum_{i}^{N}\left\langle\Delta_{d}\left(r_{i}, p, T\right)\right\rangle / N$ is the pairing potential. The quantity $R_{\mathrm{n}}(p)$ is proportional to the normal state in-plane resistivity $\rho_{a b}\left(p, T \geq T_{c}\right)$. In what follows we assume $R_{\mathrm{n}}(p)$ for the optimally-doped compounds, and in the case of $(\mathrm{Pb}, \mathrm{La})-\mathrm{Bi} 2201$ use experimental values of the in-plane resistivity ratio $\rho_{a b}(p) / \rho_{a b}(p=0.16)$ to calculate $R_{\mathrm{n}}(p)$ for the underdoped samples. Since the relationship between $T_{c} / T_{c}^{\max }$ and the hole concentration $p$ for $(\mathrm{Pb}$, $\mathrm{La})-\mathrm{Bi} 2201$ is the same as for La-doped Bi22013ㅡ, we have used available resistivity data for La-doped Bi220146 in our calculations shown in Table III For orthorhombic Y123, we instead used experimental values of the $b$-axis resistivity ratio $\rho_{b}(p) / \rho_{b}(p=0.16)$ from Ref. 47 to estimate $R_{\mathrm{n}}(p) / R_{\mathrm{n}}(p=$ $0.16)$.

As the temperature is lowered below $T^{*}$, thermal fluctuations diminish and long-range phase coherence between the individual SC domains is established when $k_{\mathrm{B}} T \approx$ $\left\langle E_{\mathrm{J}}(p, T)\right\rangle$. The temperature $T$ at which this occurs defines the bulk critical temperature $T_{c}$. Figs. 3 (a) and 3 (b) show the temperature dependence of $\left\langle E_{\mathrm{J}}(p, T)\right\rangle$ for both compounds at the different dopings. The intersection of the $\left\langle E_{\mathrm{J}}(p, T)\right\rangle$ curves with the $k_{\mathrm{B}} T$ line yields values of $T_{c}$ in good agreement with the actual values for Y123 and (Pb, La)-Bi2201 (Fig. 4 and Tables \and 
TABLE I. Experimental data for $\Delta_{\mathrm{SC}}$ and $\Delta_{\mathrm{PG}}$ determined from universal curves that describe Y123 and a number of other high- $T_{c}$ cuprate superconductors (Ref. 44). An experimental value for $\Delta_{\mathrm{SC}}$ at $p=0.12$ is omitted, since deviations from the universal curve are expected for Y123 near 1/8 hole doping, where $T_{c}$ plateaus. The value of $T^{*} \approx 278 \mathrm{~K}$ at $p=0.09$ is estimated from a linear extrapolation of data in (Ref. 50). We tune the scaling factors explained in the text to yield the blue and green values at optimum doping. Red values are calculated with the same parameters.

\begin{tabular}{|cccc|}
\hline \hline & $p=0.16$ & $p=0.12$ & $p=0.09$ \\
\hline$\lambda_{\mathrm{CO}}\left(a_{0}\right)^{4.5,7}$ & $3.49 \pm 0.16$ & $3.15 \pm 0.16$ & $3.00 \pm 0.16$ \\
$\left\langle V_{\mathrm{GL}}(\mathbf{r})\right\rangle$ & -0.156 & -0.110 & -0.105 \\
$\rho_{b}\left(1.05 T_{c}\right)(\mu \Omega \mathrm{cm})^{47}$ & $\approx 40$ & $\approx 50$ & $\approx 70$ \\
\hline$\Delta_{\mathrm{SC}}{ }^{44}(\mathrm{meV})$ & $42 \pm 2$ & - & $24 \pm 1$ \\
$\left\langle\Delta_{d}(\mathbf{r})\right\rangle(\mathrm{meV}$ & 43.8 & 25.5 & 23.3 \\
$T_{c}(\mathrm{~K})^{47.49}$ & 93.4 & 66 & 55 \\
& 92.0 & 68.1 & 55.6 \\
\hline$\Delta_{\mathrm{PG}}{ }^{44}$ & 76 & 104 & 124 \\
$T^{* 49}$ & 76 & $93.4 \pm 9$ & $103.3 \pm 9$ \\
& 170 & 232 & 278 \\
& 170 & $209 \pm 19$ & $231 \pm 21$ \\
\hline \hline
\end{tabular}

\section{THE PSEUDOGAP}

Next, we use the free-energy simulations to make a simple estimate of the PG, under the assumption that the PG appears due to the mesoscale phase separation ${ }^{26}$ that creates small domains of $\mathrm{CO}$ wavelength below $T_{\mathrm{PS}} \approx T^{*}$. For Bi2201 we consider a single-particle state bound to a two-dimensional (2-D) square box of depth $\left\langle V_{\mathrm{GL}}(\mathbf{r})\right\rangle$ and sides of length $\lambda_{\mathrm{CO}}$. For Y123 we consider a single-particle state bound to a stripelike 2-D rectangular box of depth $\left\langle V_{\mathrm{GL}}(\mathbf{r})\right\rangle$, width $\lambda_{\mathrm{CO}}$, and length equivalent to the CDW correlation length, which is much longer than $\lambda_{\mathrm{CO}}{ }^{4,5,7}$. We assume in both cases that the PG is proportional to the numerical solution of the corresponding 2-D Schrödinger equation for the ground state binding energy. The proportionality factor is estimated using experimental values of the pseudogap $\Delta_{\mathrm{PG}}$ for $\mathrm{Y} 123$ and $(\mathrm{Pb}$, La)-Bi2201 at $p=0.16$ (Tables 1 and 2), and the values of $\Delta_{\mathrm{PG}}$ are calculated for the underdoped samples using the respective values of $\left\langle V_{\mathrm{GL}}(\mathbf{r})\right\rangle$ and $\lambda_{\mathrm{CO}}$. To further assess the accuracy of the results for the underdoped samples, we convert $\Delta_{\mathrm{PG}}$ to $T^{*}$ using the experimental ratios $T^{*} / \Delta_{\mathrm{PG}}=170$ $\mathrm{K} / 76 \mathrm{meV}$ and $T^{*} / \Delta_{\mathrm{PG}}=241 \mathrm{~K} / 30 \mathrm{meV}$ for optimallydoped Y123 and (Pb, La)-Bi2201, respectively. As shown in Fig. 4 and Table In the calculated values of $T^{*}$ for $(\mathrm{Pb}, \mathrm{La})$ Bi2201 at $p=0.126$ and 0.141 agree well with measurements of the PG onset temperature for La-doped Bi220148. Reasonable agreement is also obtained between the calculated and experimental ${ }^{49}$ values of $T^{*}$ for $\mathrm{Y} 123$ at $p=0.09$ and 0.12 (Fig. 4 and Table凹). As mentioned in the introduction we presume the onset of $\mathrm{CO}$ domains at $T^{*}$ above the long range temperature $T_{\mathrm{CO}}(p)<T^{*}(p)$, a behavior that has been detected by some different experiments $3,7,9,27$.
TABLE II. Experimental data for $\Delta_{\mathrm{SC}}$ and $\Delta_{\mathrm{PG}}$ from STM with the indicated $T_{c}$ values ${ }^{11}$. The hole doping $p$ was determined from the $T_{c}$ versus $p$ relationship obtained by $\mathrm{X}$-ray absorption experiments on $(\mathrm{Pb}, \mathrm{La})-\mathrm{Bi} 2201$ and La-doped Bi2201 ${ }^{43}$. The in-plane resistivity $\rho_{\text {ab }}$ data area for La-doped $\mathrm{Bi} 2201^{46}$ and $T^{*}$ data are from intrinsic tunneling measurements ${ }^{48}$. We tune the scaling factor explained in the text to yield the blue and green values at optimum doping. Red values are calculated without any extra parameters.

\begin{tabular}{|cccc|}
\hline \hline & $p=0.16$ & $p=0.12$ & $p=0.09$ \\
\hline$\lambda_{\mathrm{CO}}\left(a_{0}\right)^{11}$ & $6.2 \pm 0.2$ & $5.1 \pm 0.2$ & $4.5 \pm 0.2$ \\
$\left\langle V_{\mathrm{GL}}(\mathbf{r})\right\rangle$ & -0.1022 & -0.1018 & -0.1021 \\
$\rho_{b}\left(1.05 T_{c}\right)(\mu \Omega \mathrm{cm})^{46}$ & 18.3 & 24.8 & 28.3 \\
\hline$\Delta_{\mathrm{SC}}{ }^{11}(\mathrm{meV})$ & 15 & - & - \\
$\left\langle\Delta_{d}(\mathbf{r})\right\rangle(\mathrm{meV}$ & 15.8 & 13.2 & 9.2 \\
$T_{c}\left(\mathrm{~K}^{11}\right.$ & 35 & 32 & 25 \\
& 35.2 & 32.5 & 24.7 \\
\hline$\Delta_{\mathrm{PG}}{ }^{12}$ & $30 \pm 12$ & $45 \pm 15$ & $68 \pm 20$ \\
$T^{* 48}$ & 30 & 44.3 & 54.2 \\
& 241 & 355 & 446 \\
& 241 & 327.9 & 427.4 \\
\hline \hline
\end{tabular}

\section{COMPARISON OF CALCULATED AND EXPERIMENTAL PARAMETERS}

Tables $\llbracket$ and $\Pi$ contain values of experimental parameters (denoted by black text) used in the calculations for each compound, and the calculated parameters (denoted by red text). For each cuprate family the calculated values at $p=0.16$ (which are denoted by blue and green text) were multiplied by a scaling factor to match experimental values as follows:

i) The proportionality constant between $\left\langle V_{\mathrm{GL}}(\mathbf{r})\right\rangle$ and the attractive pairing potential $V$ of Eq. (B4) was adjusted to yield a calculated value of $\left\langle\Delta_{d}(\mathbf{r})\right\rangle$ that approximately equals the experimental value of the $\mathrm{SC}$ gap $\Delta_{\mathrm{SC}}$ at $p=0.16$. This proportionality constant, once determined, was subsequently used for all other values of $p$.

ii) The scaling factor between the normal resistance $R_{n}$ in Eq. (3) and the resistivity $\rho_{\mathrm{b}}(1.05 T c)$ just above $T_{c}$ was adjusted until the calculated value of $T_{c}$ at $p=0.16$ approximately equaled the experimental value. This same scaling factor was used for all other values of $p$.

iii) The ground state binding energy of a single-particle in a 2-D square (rectangular) box was multiplied by a proportionality factor so as to equal the PG of (Pb, La)-Bi2201 (Y123) at $p=0.16$. Again, this same proportionality constant was used for the calculations at other dopings.

\section{FERMI ARC FORMATION}

Next we show that the phase separation approach considered above is able to reproduce the ungapped portion of the Fermi surface (Fermi arcs) that is known to occur near the nodal region just above $T_{c} \underline{51-53}$. We start by recalling that 
Figs. 1(c), 1(f), 2(c) and 2(f) show domains of SC order parameter modulations. To each domain we assign a label $j$ and a complex SC order parameter $\Delta_{d}(k, T) \exp \left(i \Phi_{j}\right)$, where $\Delta_{d}(k, T)=\Delta_{0}(T)\left[\cos \left(k_{x} a_{0}\right)-\cos \left(k_{y} a_{0}\right)\right]=$ $\Delta_{0}(T) \cos (2 \phi), \phi$ is the azimuthal angle measured from the $x$-direction in the $\mathrm{CuO}_{2}$ plane and $\Delta_{0}(T)$ is the wave function amplitude in the $\mathrm{j}^{\text {th }}$ domain at temperature $T$. The $d$ wave symmetry implies larger supercurrents flowing in the $\mathrm{CuO}_{2}$ plane along the antinodal directions parallel to the $\mathrm{Cu}-$ $\mathrm{O}$ bonds, and vanishing values along the nodal directions $\phi= \pm \pi / 4$ and $\pm \pi / 4$. The local intrinsic SC phase $\Phi_{j}$ and the superfluid density $n_{j} \propto \Delta_{d}\left(r_{j}, T\right)^{2}$ are canonically conjugate variables ${ }^{54}$, leading to large fluctuations of the phase $\Phi_{j}$ along the nodal directions, where $n_{j}$ and $\Delta n_{j}$ vanish. This is due to the quantum uncertainty principle and we may write $\Delta \Phi_{j}(\phi) \propto 1 / \cos (2 \phi)$ to indicate the azimuthal dependence of the phase uncertainty, which has its maximum and minimum values along the nodal and antinodal directions, respectively. Furthermore, $\Delta \Phi_{j}$ has a clear dependence on the Josephson coupling. In particular, as shown in the previous section, at $T<T_{c}$ all $\Phi_{j}$ are locked together leading to long range SC order, but at $T>T_{c}$ phase decoupling occurs because $\left\langle E_{\mathrm{J}}(p, T)\right\rangle<k_{\mathrm{B}} T$ and concomitantly $\Delta \Phi_{j}$ increases with $T$ up to the temperature at which $\left\langle E_{\mathrm{J}}(p, T)\right\rangle$ vanishes. In particular, $\Delta \Phi_{j}$ increases monotonically from near zero at $T_{c}$ to very large values near $T^{*}$. Furthermore, $\Delta \Phi_{j}$ has a large anisotropy when combined with the quantum effects discussed above. The two distinct contributions are separable, such that $\Delta \Phi_{j}(p, T, \phi)=\Delta \Phi_{j}(p, T) \Delta \Phi_{j}(\phi)$. We drop the index $j$ because $\Delta \Phi_{j}(\phi)$ is the same for all domains, and assume $\left\langle E_{\mathrm{J}}(p, T)\right\rangle$ is the same for all $j$. These considerations imply that just above $T_{c}$ the electrons ejected by ARPES from different domains come from regions where the SC order parameter has essentially the same $\Phi_{j}$ along the antinodal directions, and $\Delta \Phi_{j}(\phi) \approx 0$. On the other hand, such phase coherence is lost near the nodal directions where $\Delta \Phi_{j}(\phi) \approx \pi$ is maximum. Consequently, the average $\mathrm{SC}$ amplitude measured by ARPES may be written as follows ${ }^{55}$,

$$
\begin{aligned}
& \left\langle\Delta_{d}(p, T, \phi)\right\rangle= \\
& \frac{|\cos (2 \phi)|}{\Delta \Phi_{j}(p, T, \phi)} \int_{0}^{\Delta \Phi_{j}(p, T, \phi)}\langle\Delta(p, T)\rangle \cos (\Phi) d \Phi \\
& =\langle\Delta(p, T)\rangle|\cos (2 \phi)| \frac{1}{\Delta \Phi_{j}(p, T, \phi)} \sin \left[\Delta \Phi_{j}(p, T, \phi)\right] .
\end{aligned}
$$

This expression contains the two distinct contributions that weaken phase coherence, one from quantum oscillations that depends only on the azimuthal angle $\phi$ and one from thermal oscillations that competes with the average Josephson coupling, leading to $\Delta \Phi_{j}(p, T, \phi)=\Delta \Phi_{j}(p, T) \Delta \Phi_{j}(\phi)$ for all "j". We may take $\Delta \Phi(\phi) \sim 1 / \cos ^{2}(2 \phi)$, which satisfies the expected inverse cosine dependence and the square makes it symmetric and always positive around the nodal directions ( $\phi= \pm \pi / 4$ and $\pm \pi / 4$ ). Now we can infer the functional form of $\Delta \Phi(p, T)$ noting that for $T<T c$, all $\Phi_{j}$ are locked together leading to long range order and $\Delta \Phi_{j} \sim 0$. On the other hand, for $T \leq T_{c}$ all $\Phi$ decouple because $\left\langle E_{\mathrm{J}}(p, T)\right\rangle<k_{\mathrm{B}} T$ and $\Delta \Phi>0$. Above $T_{c},\left\langle E_{\mathrm{J}}(p, T)\right\rangle$ decreases with increasing $T$ and vanishes near $T^{*}$. Concomitantly $\Delta \Phi$ increases. Thus, there are three distinct temperature dependent regimes:

(i) $T \leq T_{c}$ : Since $\Delta \Phi \sim 0$, Eq. (4) is easy to solve and we obtain the "bare" expression $\left\langle\Delta_{d}(\mathbf{r}, T, \phi)\right\rangle=$ $\left\langle\Delta_{d}(p, T)\right\rangle \mid \cos (2 \phi)$.

(ii) $T>T^{*}:\langle\Delta(p, T)\rangle \sim 0$ and it is clear that there is no gap. (iii) $T_{c}\left\langle T\right.$ : Taking into account the effect of $\left\langle E_{\mathrm{J}}(p, T)\right\rangle$ we assume $\Delta \Phi(p, T)=A\left[1-\left\langle E_{\mathrm{J}}(p, T)\right\rangle / k_{\mathrm{B}} T_{c}(p)\right.$ where $A$ is a constant. This expression vanishes at $T_{c}$ and increases monotonically with $p$, as expected from ARPES experiments ${ }^{51.52}$. Thus, putting all together, $\Delta \Phi(p, T, \phi)=\left[A / \cos ^{2}(2 \phi)\right][1-$ $\left\langle E_{\mathrm{J}}(p, T)\right\rangle / k_{\mathrm{B}} T_{c}(p)$ and we obtain the value of $A$ by comparing with the onset of the measured ${ }^{51}$ gapless region for a given sample. To reproduce the measured gapless regions we also assume in Eq. (4) that $\left\langle\Delta_{d}(p, T, \phi)\right\rangle \sim 0$ whenever $\Delta \Phi(p, T, \phi) \geq \pi$, due to destructive phase interference from electrons ejected from different domains.

Specifically we use the ARPES measurements $\frac{51}{2}$ at $T=$ $102 \mathrm{~K}$ for the Bi2212 compound with $T_{c}=92 \mathrm{~K}$, which shows a gapless region between $28^{0} \leq \phi \leq 62^{0}$ to derive the constant $A$. Equating $\Delta \Phi(p \sim 0.15, T=102 \mathrm{~K}, \phi=$ $\left.28^{0}, 62^{0}\right)=\pi$, yields $\left\langle\Delta_{d}(p, T, \phi)\right\rangle \sim 0$ and this is possible if we take $A=2.84 \pi$. Note that $\phi$ is measured from the $(\pi, \pi)$ to $(0, \pi)$ direction of the Brillouin zone according to Refs. 51 and 52. With $\Delta \Phi(p, T, \phi)$ determined, we may apply the derived equation to any sample. In particular, we apply this expression to the other two Bi2212 compounds measured in Ref. 51. Some above $T_{c}$ values of $\left\langle E_{\mathrm{J}}(p, T)\right\rangle$ used in the calculations are plotted in Fig.(5) for illustration purpose. Accordingly we obtain for the $T_{c}=86 \mathrm{~K}$ compound a gapless region at $23.8^{0} \leq \phi \leq 66.2^{0}$, which compares well with the experimental $25^{0} \leq \phi \leq 65^{0}$. For the underdoped $T_{c}=75 \mathrm{~K}$ Bi2212 sample at $T=85 \mathrm{~K}$, we obtain $36.5^{0} \leq \phi \leq 53.5^{0}$, which is also in good agreement with the experimental result $\frac{51}{}$. We summarize the Fermi arc calculations for the three samples in Fig. 6, where the results of the "envelope" phase factor of $\left\langle\Delta_{d}(p, T, \phi)\right\rangle$

$$
R=\frac{|\cos (2 \phi)|}{\Delta \Phi(p, T, \phi)} \times \sin \left[\Delta \Phi_{j}(p, T, \phi)\right]
$$

from Eq. (4) are in good agreement with the experiments 51 . The arrows mark the experimentally determined onset of the gapless regions for each sample, as described above.

\section{SUMMARY AND CONCLUSIONS}

Our theory infers a fundamental link between the periodicity of the CDW and the PG and SC energy scales of hightemperature cuprate superconductors, and shows that within this framework it is possible to account for the onset temperatures $T_{c}$ and $T^{*}$ of two different cuprate families. We stress that the only quantitative assumptions made in our calculations for underdoped $\mathrm{Y} 123$ and $(\mathrm{Pb}, \mathrm{La})-\mathrm{Bi} 2201$ pertain to a natural scaling factor, which we have determined by scaling calculated free energy parameters to achieve values of the PG 


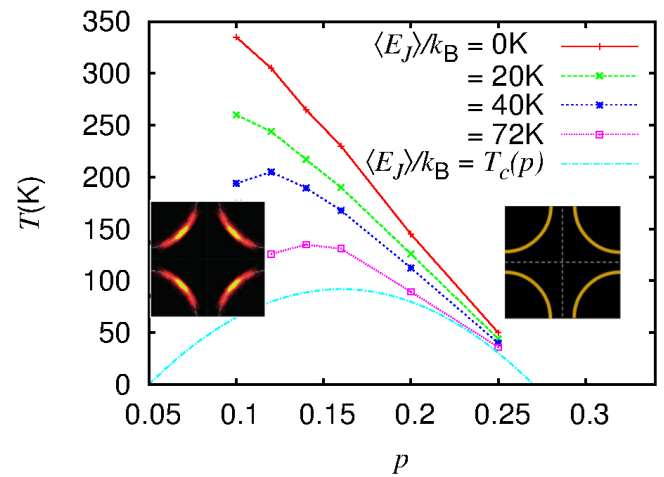

FIG. 5. Josephson coupling energy phase diagram and schematic Fermi surface. The variation of the average Josephson coupling energy with doping and temperature for Bi2212 (from Ref. 45). Above the onset of $\left\langle E_{\mathrm{J}}\right\rangle / k_{\mathrm{B}}=0 \mathrm{~K}$ there is no SC gap. The corresponding gapless Fermi surface is depicted at the right of the phase diagram. In the region of the phase diagram where $T \geq T_{c}$, the average SC gap may be finite. However, combined thermal and quantum phase fluctuations may cause destructive interference in the ARPES data along the nodal directions, leading to the gapless Fermi arcs shown in the left of the figure.

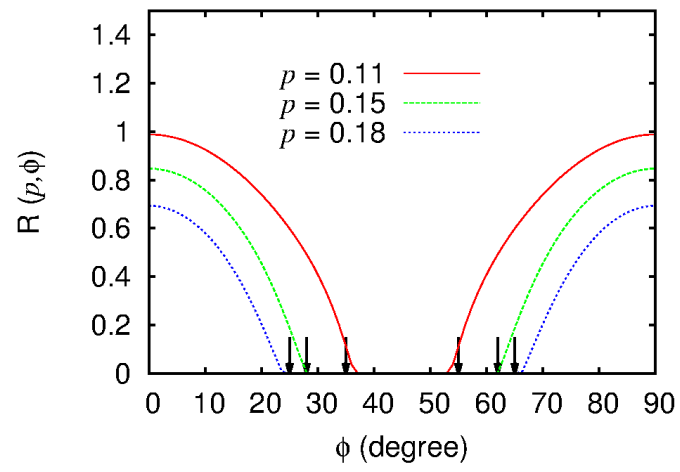

FIG. 6. The phase factor from the SC fluctuation as function of the azimuthal angle. The envelope of the SC amplitude $\left\langle\Delta_{d}(p, T, \phi)\right\rangle$ according to Eq. (4). The arrows show the limits of the gapless region as determined from ARPES experiments on Bi2212 at $T=$ $T_{c}(p)+10 \mathrm{~K}$ for three dopings ${ }^{51}$.

and SC gap that agree with experimental values at one particular doping. Our model is general in the sense that it can be applied to other cuprate families, provided the doping dependence of the CDW order is known.

Our approach generates a local free energy potential having a spatial periodicity that matches that of the experimentally observed short-range static CDW order. Our calculations in the framework of BdG theory yield different SC amplitudes in distinct charge-ordered domains that generally vanish only above $T_{c}$. Our approach is consistent with experiments $\frac{911,36,38}{1}$ that measure a finite $\mathrm{SC}$ amplitude above $T_{c}$, and promotes the scenario whereby the SC resistive transition marks the onset of global phase coherence between SC domains. In our model Fermi arcs appear above $T_{c}$ because there are large phase fluctuations along the nodal directions where the superfluid density vanishes. The increase of the arcs size with $p$ is reproduced because the dependence of $\left\langle E_{\mathrm{J}}(p, T)\right\rangle$ on the temperature changes rapidly with doping.

Finally we address the experimental observations indicating a competition between superconductivity and $\mathrm{CO}$. While $\mathrm{X}$-ray experiments show a decrease of the CDW diffraction intensity and correlation length below $T_{C} \stackrel{1,2.4 .5 .8}{\text {, these measure- }}$ ments seem to be detecting static charge correlations. Static CDW order competes with superconductivity by reducing the number of charge carriers available for pairing. On the other hand, our theory requires that dynamic $\mathrm{CO}$ is also present to induce a fluctuating hole-pair potential that scales with $V_{\mathrm{GL}}$. While there is some evidence for CDW fluctuations from optical pump-probe ${ }^{56}$ and low-energy, momentum-resolved electron energy-loss spectroscopy $\underline{\underline{50}}$ experiments, there is currently insufficient experimental information to assess the pervasiveness or significance of fluctuating $\mathrm{CO}$ in cuprates.

\section{ACKNOWLEDGMENTS}

We thank S. Ono and Y. Ando for providing us with their resistivity data. We also thank Andrea Damascelli, AndréMarie Tremblay and John Tranquada for informative discussions. Supported by the Brazilian agencies FAPERJ and CNPq (E.V.L.M.); the Canadian Institute for Advanced Research (CIFAR) and the Natural Sciences and Engineering Research Council of Canada (J.E.S.).

\section{Appendix A: CHARGE ORDER SIMULATIONS}

To describe the growth and development of spatial charge inhomogeneity in the $\mathrm{CuO}_{2}$ planes we applied a theory of phase-ordering dynamics, whereby the system evolves through domain coarsening when quenched from a homogeneous into a broken-symmetry phase. The time-dependent $\mathrm{CH}$ approach provides a simple way to determine the time evolution of the $\mathrm{CO}$ process $\stackrel{41}{ }$. The $\mathrm{CH}$ equation can be written in the form of the following continuity equation for the local free energy current density $\mathbf{J}=-M \nabla^{2}\left((\partial f / \partial u)^{57}\right.$

$$
\frac{\partial u}{\partial t}=-\nabla . \mathbf{J}=-M \nabla^{2}\left[\varepsilon^{2} \nabla^{2} u+\frac{\partial V_{\mathrm{GL}}}{\partial u}\right],
$$

where $M$ is the charge mobility that sets the phase separation time scale. The order parameter varies between $u(\mathbf{r}, t) \sim 0$ for the homogeneous system above the phase-separation onset temperature $T_{\mathrm{PS}}$, and $u(\mathbf{r}, t \rightarrow \infty)= \pm 1$ for the extreme case of complete phase separation. We solved the $\mathrm{CH}$ equation by a stable and fast finite difference scheme with free boundary conditions $\mathrm{s}^{55}$. The spatial dependence of the charge density obtained by numerically solving the $\mathrm{CH}$ equation evolves with time $t=n \delta t$, where $\mathrm{n}$ is the number of time steps $\delta t$. When the order parameter is conserved, as in phase separation, the charges can only exchange locally rather than over large distances. This leads to diffusive transport of the order parame- 
ter. Consequently, at early times or small n, we obtain charge modulations with periodicities of only a few lattice constants. Using different parameters and initial conditions we are able to reproduce the experimentally determined $\mathrm{CO}$ patterns in cuprates. Although these simulations are not the stable solutions of the $\mathrm{CH}$ equation (as is clear from the time evolution of the simulations shown in Fig. 7), the aim here is to generate periodic charge modulations with experimentally determined wavelengths that can be subsequently used to calculate the SC gap and PG in our phase- separation model. For convergence the time step $\delta t$ and spatial step $h \approx 1 / N$ for a square lattice of $N^{2}$ sites must be such that $\delta t \leq h^{2} / 9$ (Ref. 55). For the calculations here we used $\delta t \leq h^{2} / 10$ and $h=1 / 100$.

In the main paper we present detailed $\mathrm{CO}, T_{c}$ and $T^{*}$ calculations for six compounds. Three of the Bi2201 and three of the Y123 families. In the next two paragraphs we give the values of some parameter used in the $\mathrm{CH}$ simulations.

(Pb, La)-Bi2201: Simulations with $\alpha=B=1$, time steps of $n=700,900,1300$ and $\varepsilon=0.012,0.014,0.0175$ yield checkerboard $\mathrm{CO}$ patterns with the desired wavelengths $\lambda_{\mathrm{CO}}$ for $(\mathrm{Pb}, \mathrm{La})-\mathrm{Bi} 2201$ (at $p=0.126,0.141$ and 0.16 ) near $4.5 a_{0}, 5.1 a_{0}$, and $6.2 a_{0}$, respectively. The fewer time steps required to simulate the $\mathrm{CO}$ patterns of the underdoped samples is indicative of a reduced charge mobility, and is consistent with an increase of the normal-state resistivity. At later times (i.e., greater $n$ ) the periodic electronic structure evolves into an irregular patch-like system of segregated low- and highcharge density regions. In addition, the length scale of the system increases with the phase separated regions forming larger domains. This latter situation was considered previously ${ }^{45}$. Fig. 7 shows $\mathrm{CH}$ simulations of $u(\mathbf{r}, t)$ at times beyond where checkerboard $\mathrm{CO}$ with $\lambda_{\mathrm{CO}}=4.5 a_{0}$ is observed in $(\mathrm{Pb}, \mathrm{La})$ Bi2201 at $p=0.126$.

Y123: Simulations with $\alpha=1, B=5, \varepsilon=0.0053,0.0055$, 0.0058 and time steps $n=35,38,42$ yield charge stripe patterns with the desired wavevectors $Q=0.333,0.317$ and 0.287 r.l.u. $\left(\lambda_{\mathrm{CO}}=1 / Q\right)$ estimated from (Refs. 4, 5, and 7) for Y123 at $p=0.09,0.12$ and 0.16 , respectively. Note that the values of $\mathrm{n}$ are much shorter than needed to simulate the checkerboard CO patterns of (Pb, La)-Bi2201. Because of the fewer time steps, the simulations for Y123 are somewhat less sharp.

\section{Appendix B: Combined Bogoliubov-deGennes (BdG) and Cahn-Hilliard (CH) Calculations}

We performed self-consistent calculations with the BdG theory (Refs 58 and 59) for each of the $\mathrm{CH}$ simulated charge density maps (Figs. 11a) and 1(d), and Figs. 2]a) and 2(d)). To calculate the SC pairing amplitude we assumed the attractive interaction potential $V$ scales with $V_{\mathrm{GL}}^{\min } \sim$ $\left\langle V_{\mathrm{GL}}(\mathbf{r})\right\rangle$. The SC calculations begin with the extended Hubbard Hamiltonian ${ }^{58,59}$. To describe the charge carriers dynamics in the $\mathrm{CuO}_{2}$ planes of the HTSC we consider this Hamil- tonian in a square lattice

$$
\begin{aligned}
H= & -\sum_{\{i j\} \sigma} t_{i j} c_{i \sigma}^{\dagger} c_{j \sigma}+\sum_{i \sigma} \mu_{i} n_{i \sigma} \\
& +U \sum_{i} n_{i \uparrow} n_{i \downarrow}-\frac{V}{2} \sum_{\langle i j\rangle \sigma \sigma^{\prime}} n_{i \sigma} n_{j \sigma^{\prime}}
\end{aligned}
$$

where $c_{i \sigma}^{\dagger}\left(c_{i \sigma}\right)$ is the usual fermionic creation (annihilation) operator at site $i$, the spin $\sigma$ is up $\uparrow$ or down $\downarrow$. $n_{i \sigma}=c_{i \sigma}^{\dagger} c_{i \sigma}$ is the number operator, and $t_{i j}$ is the hopping between sites $i$ and $j$. $U$ is the magnitude of the on-site repulsion, and $V$ is the magnitude of the nearest-neighbor attractive interaction. $\mu_{i}$ is the local chemical potential derived in the self-consistent process through which the local charge density is calculated by the $\mathrm{CH}$ equation and is maintained fixed. For ( $\mathrm{Pb}, \mathrm{La})-\mathrm{Bi} 2201$ we used nearest-neighbor hopping $t=0.15 \mathrm{eV}$, next-nearestneighbor hopping $t_{2}=-0.27 t$, and third nearest-neighbor hopping $t_{3}=0.19 t$ derived from angle-resolved photoemission spectroscopy (ARPES) dispersion relations ${ }^{60}$. For Y123, we used the ARPES results $t=0.15 \mathrm{meV}, t_{2}=-0.50 t$ and $t_{3}=0.16 t$ (Ref. 61). The BdG mean-field equations are ${ }^{59}$

$$
\left(\begin{array}{cc}
K & \Delta_{d}(\mathbf{r}) \\
\Delta_{d}^{*}(\mathbf{r}) & -K^{*}
\end{array}\right)\left(\begin{array}{l}
u_{n}(\mathbf{r}) \\
v_{n}(\mathbf{r})
\end{array}\right)=E_{n}\left(\begin{array}{l}
u_{n}(\mathbf{r}) \\
v_{n}(\mathbf{r})
\end{array}\right)
$$

with

$$
\begin{aligned}
K u_{n}(\mathbf{r}) & =-\sum_{\mathbf{R}} t_{\mathbf{r}, \mathbf{r}+\mathbf{R}} u_{n}(\mathbf{r}+\mathbf{R})+\mu(\mathbf{r}) u_{n}(\mathbf{r}) \\
\Delta_{d} u_{n}(\mathbf{r}) & =\sum_{\mathbf{R}} \Delta_{d}(\mathbf{r}) u_{n}(\mathbf{r}+\mathbf{R})
\end{aligned}
$$

and similar equations for $v_{n}(\mathbf{r})$, where $\mathbf{r}+\mathbf{R}$ is the position of the nearest-neighbor sites, and $\mu(\mathbf{r}) \equiv \mu_{i}$ is the local chemical potential. These equations are solved numerically for eignevalues $E_{n}(\geq 0)$ self-consistently with the spatiallyvarying $d$-wave pairing potential ${ }^{58}$

$$
\begin{aligned}
\Delta_{d}(\mathbf{r})= & -\frac{V}{2} \sum_{n}\left[u_{n}(\mathbf{r}) v_{n}^{*}(\mathbf{r}+\mathbf{R})\right. \\
& \left.+v_{n}^{*}(\mathbf{r}) u_{n}(\mathbf{r}+\mathbf{R})\right] \tanh \frac{E_{n}}{2 k_{B} T},
\end{aligned}
$$

where $V=V(T, p)$ was defined in Eq. 2. The results of $\left\langle\Delta_{d}(\mathbf{r}, T)\right\rangle$ are plotted in Fig. 8 for the three compounds of the Y123 system. Concomitantly, the spatially-varying hole density of charge carriers is given by

$$
p(\mathbf{r})=1-2 \sum_{n}\left[\left|u_{n}(\mathbf{r})\right|^{2} f_{n}+\left|v_{n}(\mathbf{r})\right|^{2}\left(1-f_{n}\right)\right]
$$

where $f_{n}=\left[\exp \left(E_{n} / k_{\mathrm{B}} T+1\right]^{-1}\right.$ is the Fermi occupation function. It is important to emphasize that the spatially inhomogeneous distribution of charge generated by the $\mathrm{CH}$ equation for different dopings was kept fixed while the local chemical potential $\mu(\mathbf{r})$ was self-consistently determined in the convergence process. This procedure incorporates the charge inhomogeneity in the calculations in a natural way. 

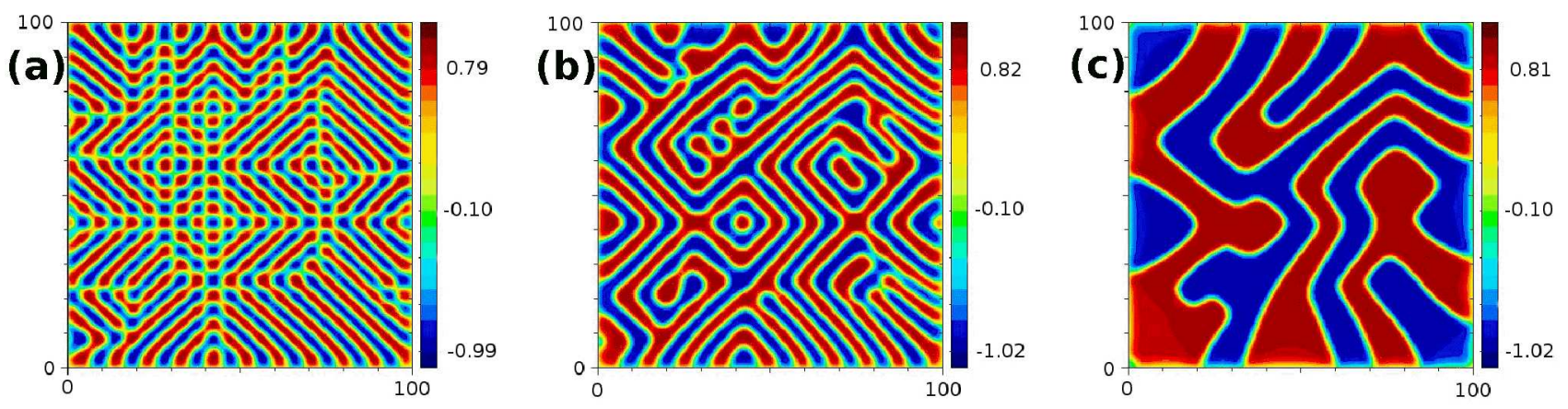

FIG. 7. Two-dimensional CH simulations of $u(r, t)$ for $\alpha=B=1, \varepsilon=0.012$ and time steps (a) $n=900$, (b) $n=1500$, and (c) $n=9000$. These plots are continuation of the time evolution of Fig. 2(a) with $n=700$.

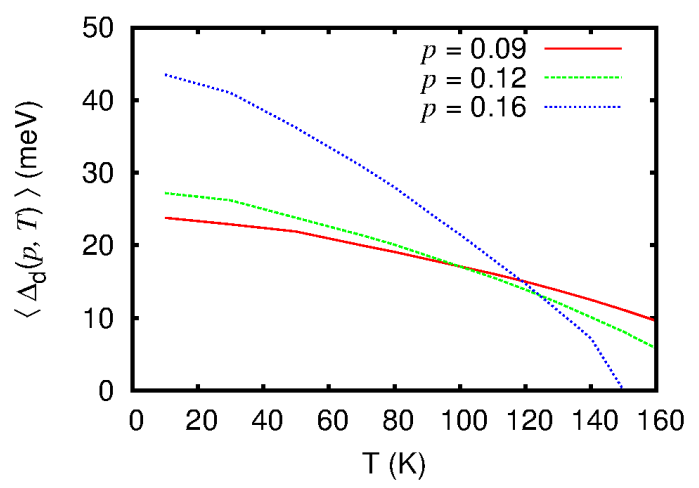

FIG. 8. Example of calculated $\left\langle\Delta_{d}(p, T)\right\rangle$ used to obtain $T_{c}$ : The average SC amplitudes for Y123 from BdG Eq. B4 used in the calculations of $\left\langle E_{\mathrm{J}}(p, T)\right\rangle$ [see Eq. 3]. The low temperature limits of $\left\langle\Delta_{d}(p, T=0)\right\rangle$ are also listed in Table $\square$

* Corresponding author: evandro@if.uff.br

1 J. Chang, E. Blackburn, T. Holmes, N. B. Christensen, J. Larsen, J. Mesot, Ruixing Liang, D. A. Bonn, W. N. Hardy, A. Watenphul, M. V. Zimmermann, E. M. Forgan, and S. M. Hayden, "Direct observation of competition between superconductivity and charge density wave orderin $\mathrm{YBa}_{2} \mathrm{Cu}_{3} \mathrm{O}_{6.67}$," Nat. Phys. 8, 871-876 (2012).

2 G. Ghiringhelli, M. Le Tacon, M. Minola, S. Blanco-Canosa, C. Mazzoli, N. B. Brookes, G. M. De Luca, A. Frano, D. G. Hawthorn, F. He, T. Loew, M. Moretti Sala, D. C. Peets, M. Salluzzo, E. Schierle, R. Sutarto, G. A. Sawatzky, E. Weschke, B. Keimer, and L. Braicovich, "Long-range incommensurate charge fluctuations in $(\mathrm{Y}, \mathrm{Nd}) \mathrm{Ba}_{2} \mathrm{Cu}_{3} \mathrm{O}_{6+\mathrm{x}}$." Science 337, 821-5 (2012).

3 R. Comin, A. Frano, M. M. Yee, Y. Yoshida, H. Eisaki, E. Schierle, E. Weschke, R. Sutarto, F. He, a Soumyanarayanan, Yang He, M. Le Tacon, I. S. Elfimov, Jennifer E Hoffman, G. A. Sawatzky, B. Keimer, and A. Damascelli, "Charge order driven by Fermi-arc instability in $\mathrm{Bi}_{2} \mathrm{Sr}_{2-\mathrm{x}} \mathrm{La}_{\mathrm{x}} \mathrm{CuO}_{6+\delta}$." Science 343, 390-2 (2014).

4 S. Blanco-Canosa, A. Frano, E. Schierle, J. Porras, T. Loew, M. Minola, M. Bluschke, E. Weschke, B. Keimer, and M. Le Tacon, "Resonant x-ray scattering study of charge-density wave correlations in $\mathrm{YBa}_{2} \mathrm{Cu}_{3} \mathrm{O}_{6+\mathrm{x}}$," Phys. Rev. B 90, 054513 (2014)

5 M. Hücker, N. B. Christensen, A. T. Holmes, E. Blackburn, E. M. Forgan, Ruixing Liang, D. A. Bonn, W. N. Hardy, O. Gutowski, M. V. Zimmermann, S. M. Hayden, and J. Chang, "Competing charge, spin, and superconducting orders in underdoped $\mathrm{YBa}_{2} \mathrm{Cu}_{3} \mathrm{O}_{\mathrm{y}}$," Phys. Rev. B 90, 1-11 (2014).

${ }^{6}$ Eduardo H. da Silva Neto, Riccardo Comin, Feizhou He, Ronny Sutarto, Yeping Jiang, Richard L. Greene, George A. Sawatzky, and Andrea Damascelli, "Charge ordering in the electron-doped superconductor $\mathrm{Nd}_{2-\mathrm{x}} \mathrm{Ce}_{\mathrm{x}} \mathrm{CuO}_{4}$," Science 347, 282-285 (2015)

7 R. Comin, R. Sutarto, E. H. da Silva Neto, L. Chauviere, R. Liang, W. N. Hardy, D. A. Bonn, F. He, G. A. Sawatzky, and A. Damascelli, "Superconductivity. Broken translational and rotational symmetry via charge stripe order in underdoped $\mathrm{YBa}_{2} \mathrm{Cu}_{3} \mathrm{O}_{6+\mathrm{y}}$." Science 347, 1335-9 (2015).

${ }^{8}$ R. Comin, R. Sutarto, F. He, E. H. da Silva Neto, L. Chauviere, A. Fraño, R. Liang, W. N. Hardy, D. A. Bonn, Y. Yoshida, H. Eisaki, A. J. Achkar, D. G. Hawthorn, B. Keimer, G. A. Sawatzky, and A. Damascelli, "Symmetry of charge order in cuprates," Nat. Mater. 14, 796-800 (2015) 
9 E. H. da Silva Neto, P. Aynajian, A. Frano, R. Comin, E. Schierle, E. Weschke, A. Gyenis, J. Wen, J. Schneeloch, Z. Xu, S. Ono, G. Gu, M. Le Tacon, and A. Yazdani, "Ubiquitous Interplay Between Charge Ordering and High-Temperature Superconductivity in Cuprates," Science 343, 393-396 (2014)

${ }^{10}$ K. Fujita, M. H. Hamidian, S. D. Edkins, C. K. Kim, Y. Kohsaka, M. Azuma, M. Takano, H. Takagi, H. Eisaki, S.-i. Uchida, A. Allais, M. J. Lawler, E.-a. Kim, S. Sachdev, and J. C. S. Davis, "Direct phase-sensitive identification of a d-form factor density wave in underdoped cuprates," Proc. Nat. Acad. Sci. , 1-7 (2014).

11 W. D. Wise, M. C. Boyer, Kamalesh Chatterjee, Takeshi Kondo, T. Takeuchi, H. Ikuta, Yayu Wang, and E. W. Hudson, "Charge density wave origin of cuprate checkerboard visualized by scanning tunneling microscopy," Nat. Phys. 4, 696-699 (2008)

12 W. D. Wise, Kamalesh Chatterjee, M. C. Boyer, Takeshi Kondo, T Takeuchi, H Ikuta, Zhijun Xu, Jinsheng Wen, G. D. $\mathrm{Gu}$, Yayu Wang, and E. W. Hudson, "Imaging nanoscale Fermi-surface variations in an inhomogeneous superconductor," Nat. Phys. 5, 213-216 (2009).

13 J. E. Hoffman, E. W. Hudson, K. M. Lang, V. Madhavan, H. Eisaki, S. Uchida, and J. C. Davis, "A Four-Unit-Cell Periodic Pattern of Quasiparticle States Surrounding Vortex Cores in $\mathrm{Bi}_{2} \mathrm{Sr}_{2} \mathrm{CaCu}_{2} \mathrm{O}_{8+\mathrm{d}}$,' Science 295, 466-469 (2002)

${ }^{14}$ C. Howald, H. Eisaki, N. Kaneko, M. Greven, and A. Kapitulnik, "Periodic density-of-states modulations in superconducting $\mathrm{Bi}_{2} \mathrm{Sr}_{2} \mathrm{CaCu}_{2} \mathrm{O}_{8+\delta}$," Phys. Rev. B 67, 014533 (2003)

15 Michael Vershinin, Shashank Misra, S. Ono, Y. Abe, Yoichi Ando, and Ali Yazdani, "Local Ordering in the Pseudogap State of the High-T c Superconductor $\mathrm{Bi}_{2} \mathrm{Sr}_{2} \mathrm{CaCu}_{2} \mathrm{O}_{8+\delta}$," Science 303, 1995-1998 (2004).

16 T. Hanaguri, C. Lupien, Y. Kohsaka, D-H Lee, M. Azuma, M. Takano, H. Takagi, and J. C. Davis, "A 'checkerboard' electronic crystal state in lightly hole-doped $\mathrm{Ca}_{2-\mathrm{x}} \mathrm{Na}_{\mathrm{x}} \mathrm{CuO}_{2} \mathrm{Cl}_{2}$." Nature 430, 1001-1005 (2004)

17 K. McElroy, D.-H. Lee, J. Hoffman, K. Lang, J. Lee, E. Hudson, H. Eisaki, S. Uchida, and J. C. Davis, "Coincidence of Checkerboard Charge Order and Antinodal State Decoherence in Strongly Underdoped Superconducting $\mathrm{Bi}_{2} \mathrm{Sr}_{2} \mathrm{CaCu}_{2} \mathrm{O}_{8+\delta}$," Phys. Rev. Lett. 94, 1-4 (2005)

18 M. H. Hamidian, S. D. Edkins, Chung Koo Kim, J. C. Davis, A. P. Mackenzie, H Eisaki, S Uchida, M. J. Lawler, E.-A. Kim, S. Sachdev, and K. Fujita, "Atomic-scale electronic structure of the cuprate d-symmetry form factor density wave state," Nat. Phys. 12, 150-156 (2016)

19 Subir Sachdev and Rolando La Placa, "Bond order in twodimensional metals with antiferromagnetic exchange interactions,"'Phys. Rev. Lett. 111, 027202 (2013)

${ }^{20}$ Efetov K. B., Meier H., and Pepin C., "Pseudogap state near a quantum critical point," Nat. Phys. 9, 442?446 (2013)

21 Lauren E Hayward, David G Hawthorn, Roger G Melko, and Subir Sachdev, "Angular fluctuations of a multicomponent order describe the pseudogap of $\mathrm{YBa}_{2} \mathrm{Cu}_{3} \mathrm{O}_{6+\mathrm{x}}$." Science 343, 1336-9 (2014)

22 C. Pépin, V. S. de Carvalho, T. Kloss, and X. Montiel, "Pseudogap, charge order, and pairing density wave at the hot spots in cuprate superconductors," Phys. Rev. B 90, 195207 (2014)

${ }^{23}$ Eduardo Fradkin, Steven A. Kivelson, and John M. Tranquada, "Colloquium: Theory of intertwined orders in high temperature superconductors," Rev. Mod. Phys. 87, 457-482 (2015)

24 Yuxuan Wang, Daniel F. Agterberg, and Andrey Chubukov, "Coexistence of charge-density-wave and pair-density-wave orders in underdoped cuprates," Phys. Rev. Lett. 114, 197001 (2015)

25 Yuxuan Wang, Daniel F. Agterberg, and Andrey Chubukov, "Interplay between pair- and charge-density-wave orders in under- doped cuprates," Phys. Rev. B 91, 115103 (2015)

${ }^{26}$ Eduardo Fradkin and Steven A. Kivelson, "Hightemperature superconductivity: Ineluctable complexity," Nat. Phys. 8, 864-866 (2012)

27 Colin V. Parker, Pegor Aynajian, Eduardo H. da Silva Neto, Aakash Pushp, Shimpei Ono, Jinsheng Wen, Zhijun Xu, Genda $\mathrm{Gu}$, and Ali Yazdani, "Fluctuating stripes at the onset of the pseudogap in the high-T(c) superconductor $\mathrm{Bi}_{2} \mathrm{Sr}_{2} \mathrm{CaCu}_{2} \mathrm{O}_{8+x}$." Nature 468, 677-680 (2010)

28 Tabis W., B. Yu, I. Bialo, M. Bluschke, T. Kolodziej, A. Kozlowski, Y. Tang, E. Weschke, B. Vignolle, M. Hepting, H. Gretarsson, R. Sutarto, F. He, M. Le Tacon, N. Bari?i?, G. Yu, and Greven M., "New insight into cuprate charge order from X-ray measurements of $\mathrm{HgBa}_{2} \mathrm{CuO}_{4+\delta}$," (2017). cond-mat.supr-con/1702.03348.

29 B. Fauqué, Y. Sidis, V. Hinkov, S. Pailhès, C. T. Lin, X. Chaud, and P. Bourges, "Magnetic order in the pseudogap phase of high$T_{C}$ superconductors," Phys. Rev. Lett. 96, 197001 (2006)

30 Li Y., Baledent V., Barisic N., Cho Y., Fauque B., Sidis Y., Yu G., Zhao X., Bourges P., and Greven M., "Unusual magnetic order in the pseudogap region of the superconductor $\mathrm{HgBa}_{2} \mathrm{CuO}_{4+\delta}$," Nature 455, $372 ? 375$ (2008)

31 Shekhter Arkady, Ramshaw B. J., Liang Ruixing, Hardy W. N., Bonn D. A., Balakirev Fedor F., McDonald Ross D., Betts Jon B., Riggs Scott C., and Migliori Albert, "Bounding the pseudogap with a line of phase transitions in $\mathrm{YBa}_{2} \mathrm{Cu}_{3} \mathrm{O}_{6+\delta}$," Nature 498, 75 ?77 (2013)

32 Chan M. K., Dorow C. J., Mangin-Thro L., Tang Y., Ge Y., Veit M. J., Yu G., Zhao X., Christianson A. D., Park J. T., Sidis Y., Steffens P., Abernathy D. L., Bourges P., and Greven M., "Commensurate antiferromagnetic excitations as a signature of the pseudogap in the tetragonal high- $\mathrm{T}_{c}$ cuprate $\mathrm{HgBa}_{2} \mathrm{CuO}_{4+\delta}$," Nat. Commun. 7, 10819 (2016)

33 Zhao L., Belvin C. A., Liang R., Bonn D. A., Hardy W. N., Armitage N. P., and Hsieh D., "A global inversion-symmetrybroken phase inside the pseudogap region of $\mathrm{YBa}_{2} \mathrm{Cu}_{3} \mathrm{O}_{\mathrm{y}}$," Nat. Phys. 13, 250?254 (2017)

34 J. Corson, R. Mallozzi, and J. Orenstein, "Vanishing of phase coherence in underdoped $\mathrm{Bi}_{2} \mathrm{Sr}_{2} \mathrm{CaCu}_{2} \mathrm{O}_{8+\mathrm{x}}$," Nature 406, 221223 (1999).

$35 \mathrm{Xu}$ Z. A., Ong N. P., Wang Y., Kakeshita T., and Uchida S., "Vortex-like excitations and the onset of superconducting phase fluctuation in underdoped $\mathrm{La}_{2-\mathrm{x}} \mathrm{Sr}_{\mathrm{x}} \mathrm{CuO}_{4}$," Nature 406, 486?488 (2000)

36 Kenjiro K Gomes, Abhay N Pasupathy, Aakash Pushp, Shimpei Ono, Yoichi Ando, and Ali Yazdani, "Visualizing pair formation on the atomic scale in the high-Tc superconductor $\mathrm{Bi}_{2} \mathrm{Sr}_{2} \mathrm{CaCu}_{2} \mathrm{O}_{8+\delta}$." Nature 447, 569-572 (2007)

37 Yang H.-B., Rameau J. D., Johnson P. D., Valla T., Tsvelik A., and $\mathrm{Gu}$ G. D., "Emergence of preformed Cooper pairs from the doped Mott insulating state in $\mathrm{Bi}_{2} \mathrm{Sr}_{2} \mathrm{CaCu}_{2} \mathrm{O}_{8+\mathrm{x}}$," Nature 456, 77?80 (2008)

38 A. Dubroka, M. Rössle, K. W. Kim, V. K. Malik, D. Munzar, D. N. Basov, A. A. Schafgans, S. J. Moon, C. T. Lin, D. Haug, V. Hinkov, B. Keimer, Th Wolf, J. G. Storey, J. L. Tallon, and C. Bernhard, "Evidence of a precursor superconducting phase at temperatures as high as $180 \mathrm{~K}$ in $\mathrm{RBa}_{2} \mathrm{Cu}_{3} \mathrm{O}_{(7-\delta)}(\mathrm{R}=\mathrm{Y}, \mathrm{Gd}, \mathrm{Eu})$ superconducting crystals from infrared spectroscopy," Phys. Rev. Lett. 106, 1-4 (2011)

${ }^{39} \mathrm{Lu} \mathrm{Li}$, Yayu Wang, M. J. Naughton, S. Ono, Yoichi Ando, and N. P. Ong, "Strongly nonlinear magnetization above $T_{c}$ in $\mathrm{Bi}_{2} \mathrm{Sr}_{2} \mathrm{CaCu}_{2} \mathrm{O}_{8+\delta}$," Europhys. Lett. 72, 451 (2005)

40 Yayu Wang, Lu Li, M. J. Naughton, G. D. Gu, S. Uchida, and N. P. Ong, "Field-Enhanced Diamagnetism in the Pseudogap State 
of the Cuprate $\mathrm{Bi}_{2} \mathrm{Sr}_{2} \mathrm{CaCu}_{2} \mathrm{O}_{8+\delta}$ Superconductor in an Intense Magnetic Field,' Phys. Rev. Lett. 95, 247002 (2005)

41 John W Cahn and John E Hilliard, "Free Energy of a Nonuniform System. I. Interfacial Free Energy," J. Chem. Phys. 28, 258 (1958)

42 Ricardo Comin and Andrea Damascelli, "Resonant XRay Scattering Studies of Charge Order in Cuprates," Annu. Rev. Cond. Matter Phys. 7, 369 (2016).

43 M. Schneider, R.-S. Unger, R. Mitdank, R. Müller, A. Krapf, S. Rogaschewski, H. Dwelk, C. Janowitz, and R. Manzke, "Evolution of the density of states at the Fermi level of $\mathrm{Bi}_{2-y} \mathrm{~Pb}_{y} \mathrm{Sr}_{2-x} \mathrm{La}_{x} \mathrm{CuO}_{6+\delta}$ and $\mathrm{Bi}_{2} \mathrm{Sr}_{2-x} \mathrm{La}_{x} \mathrm{CuO}_{6+\delta}$ cuprates with hole doping," Phys. Rev. B 72, 014504 (2005)

${ }^{44}$ S. Huefner, M. A. Hossain, A. Damascelli, and G. A. Sawatzky, "Two Gaps Make a High Temperature Superconductor?" Rep. Prog. Phys. 71, 062501 (2007)

45 E. V. L. de Mello and J. E. Sonier, "Charge segregation model for superconducting correlations in cuprates above $T_{c}$," J. Phys.: C. M. 26, 492201 (2014)

46 S. Ono and Yoichi Ando, "Evolution of the resistivity anisotropy in $\mathrm{Bi}_{2} \mathrm{Sr}_{2-x} \mathrm{La}_{x} \mathrm{CuO}_{6+\delta}$ single crystals for a wide range of hole doping," Phys. Rev. B 67, 104512 (2003)

47 Kouji Segawa and Yoichi Ando, "Transport Anomalies and the Role of Pseudogap in the $60-\mathrm{K}$ Phase of $\mathrm{YBa}_{2} \mathrm{Cu}_{3} \mathrm{O}_{7-\delta}$," Phys. Rev. Lett. 86, 4907-4910 (2001)

48 A. Yurgens, D. Winkler, T. Claeson, S. Ono, and Yoichi Ando, "Intrinsic Tunneling Spectra of $\mathrm{Bi}_{2}\left(\mathrm{Sr}_{2-x} \mathrm{La}_{x}\right) \mathrm{CuO}_{6+\delta}$," Phys. Rev. Lett. 90, 147005 (2003)

49 R. Daou, J. Chang, David LeBoeuf, Olivier Cyr-Choinière, Francis Laliberté, Nicolas Doiron-Leyraud, B. J. Ramshaw, Ruixing Liang, D. A. Bonn, W. N. Hardy, and Louis Taillefer, "Broken rotational symmetry in the pseudogap phase of a high- $T_{c}$ superconductor,' Nature 463, 519-522 (2010)

50 Sean Vig, Anshul Kogar, Vivek Mishra, Luc Venema, Melinda S. Rak, Ali A. Husain, Peter D. Johnson, Genda D. Gu, Eduardo Fradkin, Michael R. Norman, and Peter Abbamonte, "Fluctuating charge order in the optimally doped high temperature superconductor $\mathrm{Bi}_{2} \mathrm{Sr}_{2} \mathrm{CaCu}_{2} \mathrm{O}_{8+\mathrm{x}}$," (2016). cond-mat.supr-con/1509.04230v2

51 W. S. Lee, K. Vishik, I. M .and Tanaka, D. H. Lu, T. Sasagawa, N. Nagaosa, T. P. Devereaux, Z. Hussain, and Z-X. Shen, "Abrupt onset of a second energy gap at the superconducting transition of underdoped Bi2212." Nature 450, 81-84 (2007).

52 Teppei Yoshida, Makoto Hashimoto, Inna M. Vishik, ZhiXun Shen, and Atsushi Fujimori, "Pseudogap, Superconducting Gap, and Fermi Arc in High- $T_{c}$ Cuprates Revealed by Angle-Resolved Photoemission Spectroscopy," J. Phys. Soc. Jpn 81, 011006 (2012)

53 Keimer B., Kivelson S. A., Norman M. R., Uchida S., and Zaanen J., "From quantum matter to high-temperature superconductivity in copper oxides," Nature 518, 179?186 (2015)

54 B. I. Spivak and S. A. Kivelson, "Negative local superfluid densities: The difference between dirty superconductors and dirty Bose liquids," Phys. Rev. B 43, 3740-3743 (1991).

55 E. V. L. de Mello, "Disordered-based theory of pseudogap, superconducting gap, and Fermi arc of cuprates," Europhys. Lett. 99, 37003 (2012)

56 Darius H. Torchinsky, Fahad Mahmood, Anthony T. Bollinger, Ivan Božović, and Nuh Gedik, "Fluctuating charge-density waves in a cuprate superconductor," Nat. Mater. 12, 387-391 (2013).

57 A.J. Bray, "Theory of phase-ordering kinetics," Advances in Physics 43, 357-459 (1994)

58 E. V. L. de Mello and E. S. Caixeiro, "Effects of phase separation in the cuprate superconductors," Phys. Rev. B 70, 224517 (2004)

59 E V L de Mello, R B Kasal, and C A C Passos, "Electronic phase separation transition as the origin of the superconductivity and pseudogap phase of cuprates," J. Phys.: C. M. 21, 235701 (2009)

60 M. R. Norman, M. Randeria, H. Ding, and J. C. Campuzano, "Phenomenological models for the gap anisotropy of $\mathrm{Bi}_{2} \mathrm{Sr}_{2} \mathrm{CaCu}_{2} \mathrm{O}_{8}$ as measured by angle-resolved photoemission spectroscopy,' Phys. Rev. B 52, 615-622 (1995)

61 Matthias C. Schabel, C.-H. Park, A. Matsuura, Z.-X. Shen, D. A. Bonn, Ruixing Liang, and W. N. Hardy, "Angleresolved photoemission on untwinned $\mathrm{YBa}_{2} \mathrm{Cu}_{3} \mathrm{O}_{6.95}$. I. Electronic structure and dispersion relations of surface and bulk bands," Phys. Rev. B 57, 6090-6106 (1998) 